\title{
THERMAL ANNEALING OF InGaN/GaN STRAINED-LAYER QUANTUM WELL
}

\author{
Michael C.Y. Chan, ${ }^{*}$ Kwok-On Tsang, ${ }^{*}$ E. Herbert Li, ${ }^{*}$ and Steven P. Denbaars** \\ *Department of Electrical \& Electronic Engineering, University of Hong Kong, Pokfulam, Hong \\ Kong, ehli@eee.hku.hk \\ **Eectrical and Computer Engineering and Materials Departments, University of California, \\ Santa Barbara, CA 93106
}

\section{Cite this article as : MRS Internet J. Nitride Semicond. Res. 4S1, G6.25 (1999)}

\begin{abstract}
Quantum well (QW) material engineering has attracted a considerable amount of interest from many people because of its ability to produce a number of optoelectronic devices. QW composition intermixing is a thermal induced interdiffusion of the constituent atoms through the hetero-interface. The intermixing process is an attractive way to achieve the modification of the QW band structure. It is known that the band structure is a fundamental determinant for such electronic and optical properties of materials as the optical gain, the refractive index and the absorption. During the process, the as-grown square-QW compositional profile is modified to a graded profile, thereby altering the confinement profile and the subband structure in the QW. The blue-shifting of the wavelength in the intermixed QW structure is found in this process.

In recent years, III-nitride semiconductors have attracted much attention. This is mainly due to their large bandgap range from $1.89 \mathrm{eV}$ (wurtzite $\mathrm{InN}$ ) to $3.44 \mathrm{eV}$ (wurtzite $\mathrm{GaN}$ ). InGaN/GaN quantum well structures have been used to achieve high lumens blue and green light emitting diodes. Such structures also facilitate the production of full colour LED displays by complementing the colour spectrum of available LEDs.

In this paper, the effects of thermal annealing on the strained-layer InGaN/GaN QW will be presented. The effects of intermixing on the confinement potential of InGaN/GaN QWs have been theoretically analysed, with sublattices interdiffusion as the basis. This process is described by Fick's law, with constant diffusion coefficients in both the well and the barrier layers. The diffusion coefficients depend on the annealing temperature, time and the activation energy of constituent atoms. The optical properties of intermixed InGaN/GaN QW structure of different interdiffusion rates have been theoretically analyzed for applications of novel optical devices. The photoluminescence studies and the intermixed QW modeling have been used to understand the effects of intermixing.
\end{abstract}

\section{INTRODUCTION}

Wide-bandgap semiconductors such as GaN, AlN, InN and their ternary compounds have been studied so as to realize the semiconductor blue-green laser. As a matter of fact, the demonstration of high-brightness blue-green light-emitting diodes from the wurtzite GaN semiconductor has stimulated a lot of research on the fabrication of blue-green laser diodes using nitride-based semiconductors [1-3]. InGaN/GaN single-quantum-well structures have been used to achieve high-power blue and green light-emitting diodes. Also, room temperature pulsed lasing of a strained InGaN/GaN multiquantum-well laser diode has recently been demonstrated.

The nature crystal structure of most of the group III nitrides is wurtzite. Strain is also present in most of the group III nitrides because of the lack of substrate materials with a matched 
lattice constant and a thermal expansion coefficient. Indeed, fundamental studies of the effects of strain on wurtzite band structure play an important role in understanding the electronic and optical properties of $\mathrm{GaN}$-based optoelectronic materials and devices.

In order to tune the interesting wavelength, the concept of band-gap engineering is a useful tool to accept the particular devices operation [4]. Basically, intermixing process is one of easy way to achieve the modification of bandstructure. The intermixing process involves the interdiffusion of the constituent atoms of the heterostructure, the process temperature, and time. During the process the as-grown square-QW compositional profile is modified to a graded profile thereby altering the confinement profile and subband structure in the QW. The interdiffusion of InGaN/GaN QW system is more complex compared with $\mathrm{AlGaAs} / \mathrm{GaAs}$ and $\mathrm{InGaAs} / \mathrm{GaAs}$ because of the immiscibility of $\mathrm{InN}$ and $\mathrm{GaN}$ [5].

\section{THEORETICAL MODEL}

This paper presents a theoretical study of the effects of interdiffusion on a $25 \AA$ well/70 barrier of an $\mathrm{In}_{0.2} \mathrm{Ga}_{0.8} \mathrm{~N} / \mathrm{GaN}$ MQW structure, whose properties leads to the shift in spontaneous emission rate. The effects of interdiffusion on the strains, the splitting of the heavy-hole (HH), the light-hole $(\mathrm{LH})$, the crystal-field split-hole $(\mathrm{CH})$ subbands, the conduction band $(\mathrm{CB})$ and their dispersions, are calculated theoretically, based on the Hamiltonian derived using the multiband k•p model by Chuang and Chang [6].

The QW composition intermixing refer to the thermal induced interdiffusion of the constituent atoms through the hetero-interface. In fact, the interdiffused QW (DFQW) structures created by both impurity induced and impurity-free vacancy promoted processes have recently attracted much attention [7-9]. The interdiffusion mechanism can be a single-phase diffusion for two constituent atoms or it can be a two-phase or multiple-phase one and/or for multiple species. In InGaN/GaN DFQW structures, only the interdiffusion of group-III atoms, i.e. In and Ga atoms, occurs as there is no $\mathrm{N}$ concentration gradient across the interface. The diffusion of group III atoms in the QW structure is usually described by the Fick's law with constant diffusion coefficients in both the well and the barrier layers. The composition profile after the interdiffusion is characterized by a diffusion length $L_{d}$ defined as $L_{d}=\sqrt{ }(D t)$, where $D$ is the diffusion coefficient and $t$ is the annealing time of thermal processing. The In mole fraction across the InGaN/GaN DFQW structure after the interdiffusion is given by:

$$
x_{I n}(z)=\frac{x_{0}}{2}\left[\operatorname{erf}\left(\frac{L_{w}+2 z}{4 L_{d}}\right)+\operatorname{erf}\left(\frac{L_{w}-2 z}{4 L_{d}}\right)\right],
$$

where $\mathrm{z}$ denotes the co-ordinate along the crystal growth direction, $\mathrm{L}_{\mathrm{w}}$ is the as-grown well width and $\mathrm{x}_{0}$ is the as-grown In mole fraction.

To calculate the electron and hole wave functions in QW's, we use the multiband effective mass theory. It is a good approximation that the conduction and valence bands are decoupled. A parabolic band model and the Luttinger-Kohn Hamiltonian with strain components are used for the conduction and valence bands respectively. The envelope function scheme is adopted to describe the slowly varying part of the wavefunction. The effects of the quantum well confinement potential on the energies and envelope functions of the electron and hole subband edge at the zone centre of the Brillouin zone can be calculated separately, according to the BenDaniel and Duke model, using the one-dimensional Schrodinger-like equation that follows: 


$$
-\frac{\hbar^{2}}{2} \frac{d}{d z}\left[\frac{1}{m_{\perp r}^{*}(z)} \frac{d \psi_{r l}(z)}{d z}\right]+U_{r}(z) \cdot \psi_{r l}(z)=E_{r l} \psi_{r l}(z)
$$

where $\psi_{\mathrm{rl}}(\mathrm{z})$ is the envelope function of the $\mathrm{1}^{\text {th }}$ subband for electrons $(\mathrm{r}=\mathrm{c})$ or holes $(\mathrm{r}=\mathrm{hh}$, lh for heavy hole and light holes respectively); $\mathrm{m}_{\perp r}{ }^{*}(\mathrm{z})$ is the corresponding carrier effective mass in the $\mathrm{z}$ direction; $\mathrm{E}_{\mathrm{rl}}$ is the subband-edge energy. $\mathrm{U}_{\mathrm{r}}(\mathrm{z})$ is the confinement potential of the quantum well. Equation (2) is solved numerically using a finite difference method with the corresponding confinement profile. The strain of InGaN/GaN DFQW is compressive in nature, pushes the valence subbands downwards and further increases the bandgap.

The in-plane valence band dispersion of the wurtzite-InGaN/GaN QW is calculated using the parameters of $\mathrm{GaN}, \mathrm{InN}$ as listed in Table I. Meanwhile, alloy properties of $\operatorname{In}_{\mathrm{x}} \mathrm{Ga}_{1-\mathrm{x}} \mathrm{N}$ are obtained by linear interpolation, except for the bandgap energy, in which a bowing parameter of $\mathrm{b}=1.8 \mathrm{eV}$ for $\mathrm{InGaN} / \mathrm{GaN}$ is used [10]. The partition ratio for the band edge discontinuity at the heterojunction for the valence and conduction bands is assumed to be 33:67 for both QW systems.

Table I. Material Parameters used in calculating the band structure of InGaN/GaN QW.

\begin{tabular}{|c|c|c|}
\hline Parameters & GaN & InN \\
\hline $\mathrm{a}(\AA)$ & 3.190 & 3.544 \\
\hline $\mathrm{E}_{\mathrm{g}}(\mathrm{eV})$ at $300 \mathrm{~K}$ & 3.44 & 1.89 \\
\hline$\Delta_{1}=\Delta_{\mathrm{cr}}(\mathrm{meV})$ & 10 & 27.8 \\
\hline$\Delta_{2}=\Delta_{3}(\mathrm{meV})$ & 5.17 & 3.9 \\
\hline $\mathrm{m}_{\mathrm{e}}{ }^{\mathrm{z}}\left(\mathrm{m}_{\mathrm{o}}\right)$ & 0.2 & 0.11 \\
\hline $\mathrm{m}_{\mathrm{e}}{ }^{\mathrm{t}}\left(\mathrm{m}_{\mathrm{o}}\right)$ & 0.18 & 0.1 \\
\hline $\mathrm{A}_{1}$ & -6.56 & -9.28 \\
\hline $\mathrm{A}_{2}$ & -0.91 & -0.6 \\
\hline $\mathrm{A}_{3}$ & 5.65 & 8.68 \\
\hline $\mathrm{A}_{4}$ & -2.83 & -4.34 \\
\hline $\mathrm{A}_{5}$ & -3.13 & -4.32 \\
\hline $\mathrm{A}_{6}$ & -4.86 & -6.08 \\
\hline $\mathrm{a}_{\mathrm{c}}(\mathrm{eV})$ & -11.8 & -1.2 \\
\hline $\mathrm{D}_{1}(\mathrm{eV})$ & 0.7 & 0.35 \\
\hline $\mathrm{D}_{2}(\mathrm{eV})$ & 2.1 & 1.05 \\
\hline $\mathrm{D}_{3}(\mathrm{eV})$ & 1.4 & 1.32 \\
\hline $\mathrm{D}_{4}(\mathrm{eV})$ & -0.7 & -0.74 \\
\hline $\mathrm{C}_{13}\left(10^{11} \mathrm{dyn} \mathrm{cm}{ }^{-2}\right)$ & 10 & 9.4 \\
\hline $\mathrm{C}_{33}\left(10^{11} \mathrm{dyn} \mathrm{cm}{ }^{-2}\right)$ & 39.2 & 20 \\
\hline & & \\
\hline
\end{tabular}

We assume that the injected carrier density is $1 \times 10^{13} \mathrm{~cm}^{-2}$ in the InGaN-GaN DFQW. The spontaneous emission rate is calculated by integrating the emission rate over all the electrons and holes recombination processes. The spontaneous emission is given by the following equation:

$$
r_{s p}(E)=\frac{q^{2} n_{r} \omega}{\pi \hbar c^{3} \varepsilon_{0} m_{0}^{2} L_{w}} \cdot \int d k\left|P_{p q}\left(k_{/ \prime}\right)\right|^{2} \times f^{e}\left(E_{p}^{e}\left(k_{/ /}\right)\right)\left[1-f^{h}\left(E_{q}^{h}\left(k_{/ /}\right)\right)\right],
$$


where $q$ is the electric charge, $n_{r}$ is the refractive index, $\varepsilon_{0}$ is the dielectric constant of the vacuum, $\mathrm{c}$ is the speed of light, $\mathrm{L}_{\mathrm{w}}$ is the width of the quantum well, $\mathrm{E}$ is the photon energy, $\mathrm{P}_{\mathrm{pq}}$ is the optical matrix element, and $\mathrm{f}^{\mathrm{e}}$ and $\mathrm{f}^{\mathrm{h}}$ are the Fermi distribution functions for electrons in the conduction subband and holes in the valence subband respectively. To include the spectral broadening of each transition, the total spontaneous emission spectrum in a single DFQW structure is obtained using some spectral lineshape function over all transition energies E'. Such function is given by:

$$
R_{s p}(E)=\int d E^{\prime} \sum_{p, q} r_{p q}(E) \mathrm{L}\left(E-E^{\prime}\right)
$$

where $\mathrm{L}\left(\mathrm{E}^{-\mathrm{E}^{\prime}}\right)$ is a Lorentzian line-broadening function given by

$$
\mathrm{L}\left(E-E^{\prime}\right)=\frac{1}{\pi} \frac{\hbar / \tau_{i n}}{\left(E^{\prime}-E\right)^{2}+\left(\hbar / \tau_{i n}\right)^{2}}
$$

where $\tau_{\text {in }}$ is the intraband relaxation time. Then, the spontaneous emission spectrum can be obtained.

The spontaneous emission spectrum of the InGaN/GaN DFQW is shown in Fig.1. The light emission can shift the photon energy from $2.96 \mathrm{eV}$ to $3.02 \mathrm{eV}$ within the range of $\mathrm{L}_{\mathrm{d}}=0-4 \AA$. The results show that a magnitude of spontaneous emission enhancement of more than 5\%. The InGaN/GaN DFQW can be used in the blue LEDs, which can adjust the wavelength of the emitted photons according to the extent of intermixing.

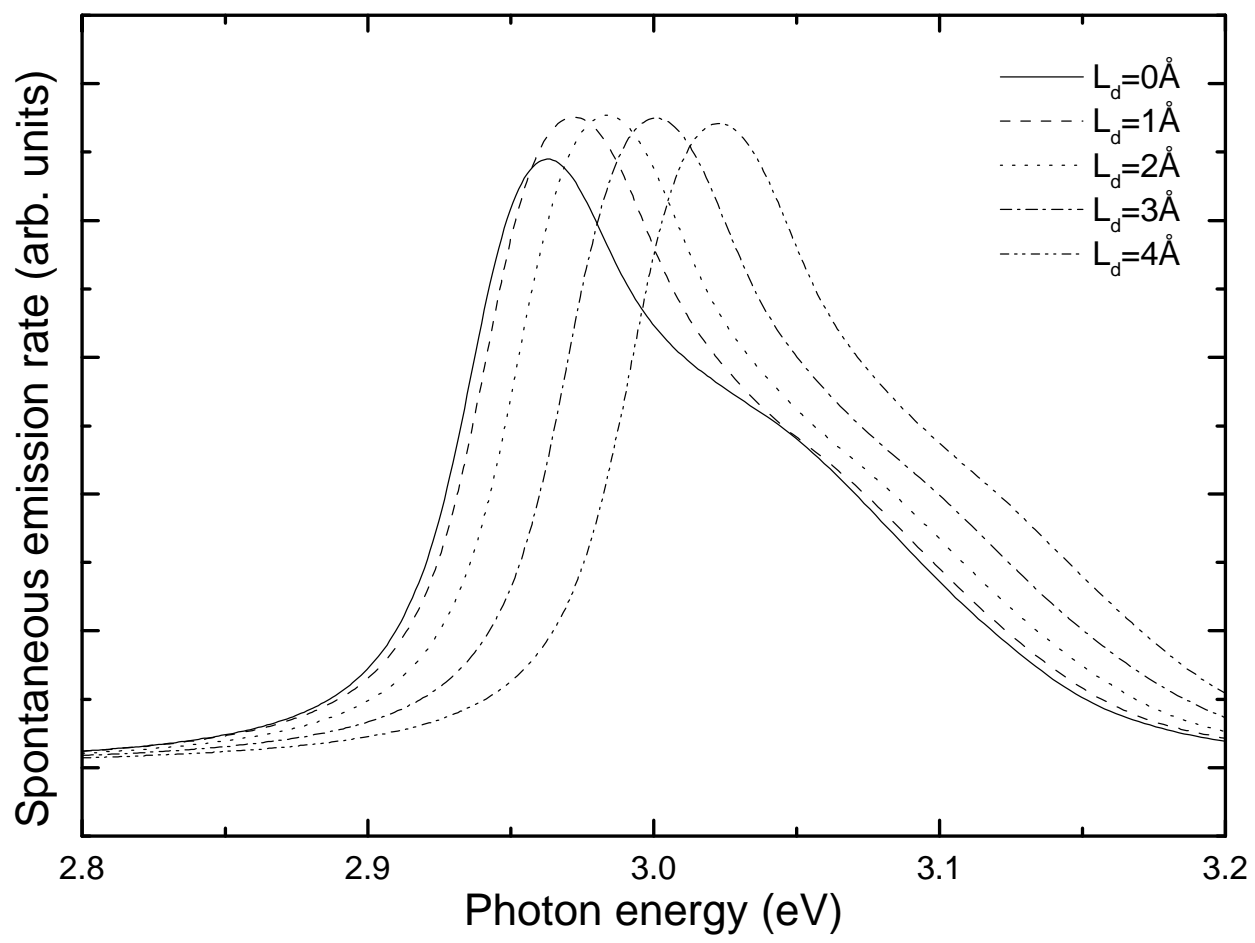


Fig.1 The spontaneous emission spectra of InGaN/GaN DFQW

\section{EXPERIMENT}

The InGaN/GaN MQW in this study were grown on c-plane sapphire substrates in an atmospheric pressure MOCVD reactor. The MQW structure consist of a $570 \AA$ GaN layer, a 10 period superlattice of $25 \AA \mathrm{In}_{0.2} \mathrm{Ga}_{0.8} \mathrm{~N}$ well/70 $\mathrm{In}_{0.05} \mathrm{Ga}_{0.95} \mathrm{~N}$ barrier, and a $2300 \mathrm{~nm}$ GaN:Si layer on a sapphire substrate. The $\mathrm{SiO}_{2}$ film was deposited on the sample surface using plasma enhanced chemical vapor deposition. Thermal annealing treatment was performed in a flowing $\mathrm{N}_{2}$ ambient using the diffusion furnace at $1060^{\circ} \mathrm{C}$ for 60 minutes. Photoluminescence spectra were taken with the $325 \mathrm{~nm}$ line of a $15 \mathrm{~mW}$ He-Cd laser at $10 \mathrm{~K}$. The PL spectra of the as-grown and intermixed MQW are shown in Fig. 2. The spectrum of the as-grown MQW is dominated by the intense localized exciton $(2.950 \mathrm{eV})$ related to the very strong blue luminescence. After the thermal annealing, there are six weak luminescence peaks in the region of $2.955-3.45 \mathrm{eV}$. The transition line (at $2.955 \mathrm{eV}$ ) for the InGaN/GaN MQW is due to the localized exciton transition from the InGaN well region and the first quantized level of exciton localization located at $3.197 \mathrm{eV}$. Two emission peaks, at approximately 3.162 and $3.071 \mathrm{eV}$, correspond to the LO phonon replicas ( 1 and $2 \mathrm{LO}$ ) of the main emission line at $3.251 \mathrm{eV}$ from the GaN layer. The energy positions are separated by $90 \mathrm{meV}$ which is close to the longitudinal optical (LO) phonon energy of bulk InN (86meV) and GaN (91meV) [11]. Also, the GaN layer is observed at 3.45eV. The energy shift of localized excitons for the as-grown and the intermixed InGaN/GaN MQW is only observed to be $5 \mathrm{meV}$. The effect of impurities enhanced intermixing is required to increase the rate of diffusion in the system.

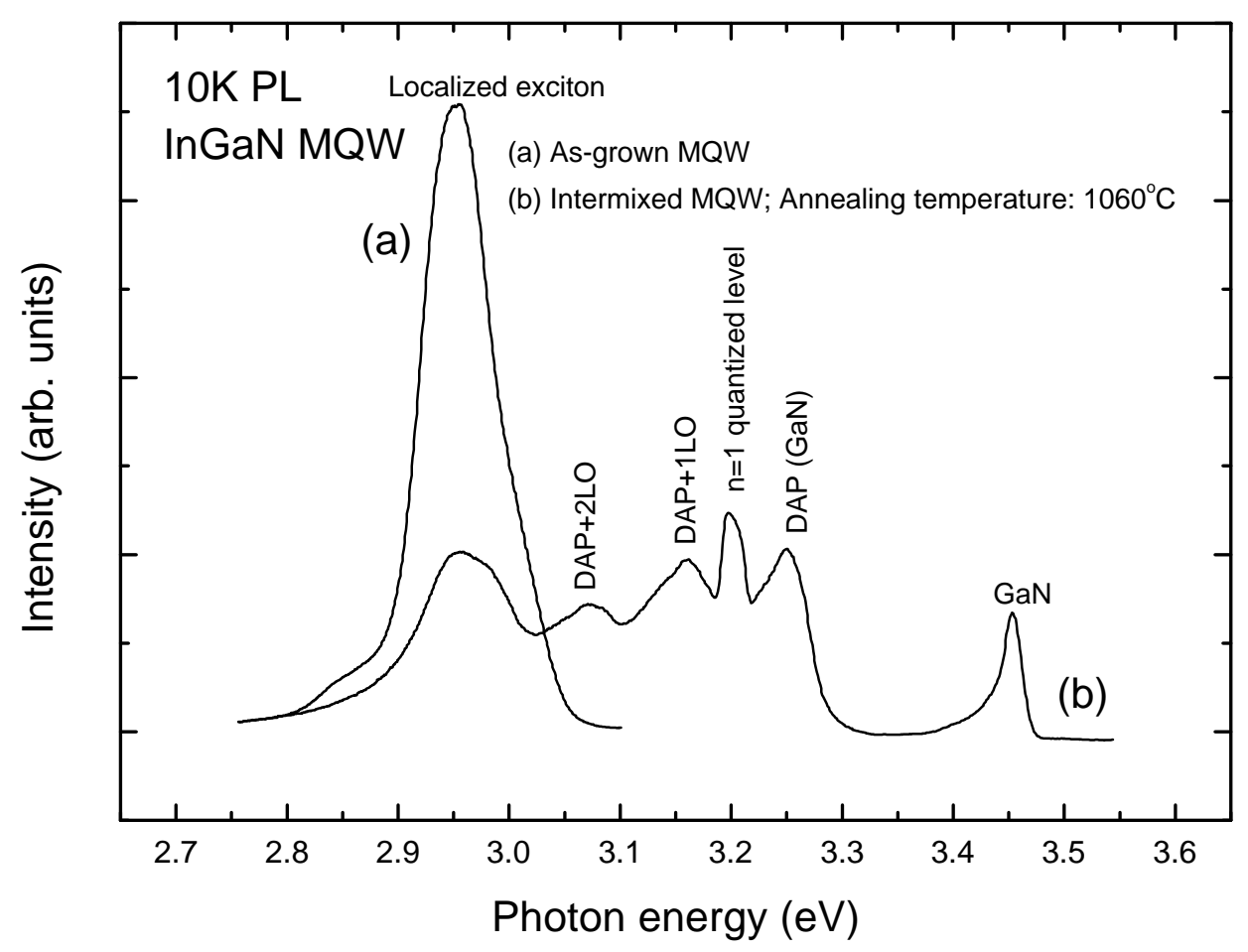

Fig.2 10K PL spectra of (a) the as-grown $\operatorname{In}_{0.2} \mathrm{Ga}_{0.8} \mathrm{~N}$ MQW and (b) the intermixed $\operatorname{In}_{0.2} \mathrm{Ga}_{0.8} \mathrm{~N}$ $\mathrm{MQW}$ at $1060^{\circ} \mathrm{C}$ for 60 minutes 


\section{CONCLUSIONS}

In this paper, the InGaN/GaN MQW intermixed structure is analyzed, and used to tune the operation wavelength of light emission in the device. Theoretically, the results show that the photon energy can be shifted from 2.96 to $3.02 \mathrm{eV}$ within the range of $\mathrm{L}_{\mathrm{d}}=0-4 \AA$ diffusion length, resulting in an enhancement of the spontaneous light emission. Experimentally, the PL of the intermixed MQW obtained the energy shifts from $2.95 \mathrm{eV}$ to $2.955 \mathrm{eV}$ ( $5 \mathrm{meV}$ blue-shift).

\section{ACKNOWLEDGEMENTS}

This work is supported by HKU-CRCG Grants. The authors would like to thank Dr. K.W. Cheah, Hong Kong Baptist University, for helping with the PL measurements, and Dr. C. Jagadish and Dr. H. H. Tan, Australian National University, for helping with the deposition of $\mathrm{SiO}_{2}$ film.

\section{REFERENCES}

1. S. Nakamura, IEEE J. Selected Topics Quantum Electron. 3, 712 (1997).

2. M. P. Mack, A. C. Abare, P. Kozodoy, M. Hanson, S. Keller, U. K Mishra, L. A. Coldren, S. P. Denbaars, institute of physics conference series, 156, 367 (1998)

3. M. P. Mack, A. C. Abare, M. Hansen, P. Kozodoy, S. Keller, U. Mishra, L. A. Coldren, S. P. Denbaars, J. of crystal growth, 190, 837 (1998)

4. E.H. Li, Editor, Quantum Well Mixing and Optoelectronic Device Applications, Milestone Series, vol. 145.

5. M. D. McCluskey, L. T. Romano, B. S. Krusor, N. M. Johnson, T.Suski, and J. Jun, Appl. Phys. Lett. 73, 1281 (1998)

6. S. L. Chuang and C. S. Chang, Semicond. Sci. Technol. 12, 252 (1997).

7. S. Yuan, Y. Kim, C. Jagadish, P. T. Burke, M. Gal, J. Zou, D. Q. Cai, D. J. H. Cockayne, and R. M. Cohen, Appl. Phys. Lett. 70, 1269 (1997).

8. S. Burkner, M. Maier, E. C. Larkins, W. Rothemound, E. P. O'Reilly, and J. D. Ralston, J. Electronic Materials 24, 805 (1995).

9. Gontijo, T. Krauss, J. H. Marsh, and R. M. De La Rue, IEEE J. Quantum Electron. 30, 189 (1994).

10. Walter R. L. Lambrecht, Solid-State Electron. 41, 195 (1997).

11. M. Smith, J. Y. Lin, H. X. Jiang, A. Khan, Q. Chen, A. Salvador, A. Botchkarev, W. Kim, and H. Morkoc, Appl. Phys. Lett. 70, 2882 (1997) 